\title{
Necesidades de formación detectadas por enfermeras de una unidad de cuidados intensivos: un estudio fenomenológico
}

\author{
Yeray G. Santana-Padilla (RN, MsC) $)^{a, *}$, L. Santana-Cabrera (MD, PhD) ${ }^{a}$, \\ M.D. Bernat-Adell (RN, MsC, PhD) $)^{b}$, T. Linares-Pérez (RN) ${ }^{c}$, \\ J. Alemán-González (RN, MsC) y ${ }^{a}$ R.F. Acosta-Rodríguez (RN, MsC) ${ }^{a}$ \\ a Hospital Universitario Insular de Gran Canaria, Las Palmas de Gran Canaria, España \\ b Universitat Jaume I de Castellón, Castellón de la Plana, España \\ c Centro de Salud Cueva Torres, Gerencia de Atención Primaria de Gran Canaria, Las Palmas de Gran Canaria, España
}

Recibido el 9 de enero de 2019; aceptado el 12 de mayo de 2019

Disponible en Internet el 3 de septiembre de 2019

\section{PALABRAS CLAVE \\ Cuidados críticos; Educación basada en competencias; Educación en Enfermería; Enfermeras clínicas}

\begin{abstract}
Resumen
Introducción: Las competencias que abordan en su entorno laboral las enfermeras de cuidados intensivos $(\mathrm{UCl})$, han aumentado con la adquisición de nuevas responsabilidades asociadas a cuidados y dispositivos a realizar al paciente crítico. Múltiples estudios avalan la necesidad de la especialización de las enfermeras que trabajan en este tipo de unidades. Apoyado en estas evidencias, la European Federation of Critical Care Nurse, recomienda unificar la formación de las enfermeras de cuidados intensivos. Por tanto, nos planteamos el siguiente objetivo: valorar las necesidades formativas que detectan las enfermeras de $\mathrm{UCl}$ a través de sus vivencias y experiencias profesionales.

Método: Estudio cualitativo descriptivo, con enfoque fenomenológico, a través de una entrevista semiestructurada donde se estudiaron los 4 ámbitos que la European Federation of Critical Care Nurse recoge (clínico, profesional, gestión y educativo). Se entrevistaron a 15 enfermeras de una UCI polivalente.

Resultados: Los entrevistados reconocen que la formación previa era deficiente para los cuidados y medidas de soporte que tuvieron que afrontar. Consideran que la formación posterior y la experiencia fueron determinantes para poder desarrollar efectivamente su labor profesional. Además afirman que las medidas de soporte y los cuidados son temas a desarrollar continuamente mediante una formación dirigida.
\end{abstract}

\footnotetext{
* Autor para correspondencia.

Correo electrónico: yeraysantana@celp.es (Y.G. Santana-Padilla).
} 
Conclusión: Las enfermeras reconocen que debe existir una formación destinada a cumplir las competencias que las $\mathrm{UCI}$ requieren, y que estas se ven afectadas por el tipo de unidad y el tipo de pacientes atendidos.

() 2019 Sociedad Española de Enfermería Intensiva y Unidades Coronarias (SEEIUC). Publicado por Elsevier España, S.L.U. Todos los derechos reservados.

\section{KEYWORDS}

Critical care; Competency-based education; Nursing Education; Nurse clinicians

\section{Training needs detected by nurses in an intensive care unit: a phenomenological} study

\begin{abstract}
Introduction: The competences of intensive care (ICU) nurses in their healthcare environment, have increased with the acquisition of new responsibilities associated with new care and devices for critical patients. Many studies suggest the need for specific training of nurses that work in these units. Based on this evidence, the European Federation of Critical Care Nurses Associations, recommends unifying the training of intensive care nurses. Therefore we set ourselves the following objective: to assess the training needs detected by ICU nurses through their experience and practical knowledge.

Method: Descriptive qualitative study, with a phenomenological approach, through semistructured interview where the four areas (clinical practice, professional, management and educational) covered by the European Federation of Critical Care Nurses Associations were studied. Fifteen nurses from an adult polyvalent ICU were interviewed.

Results: The interviewees acknowledged that the previous training was deficient for the care and support measures that they had to face. They considered that subsequent training and experience were decisive in order to carry out their work effectively. They also stated that support measures and care are topics to be developed continuously through targeted training. Conclusion: The nurses in this research study acknowledged that training is needed to achieve the competences required in ICU, and these are affected by the type of unit and patients.

(c) 2019 Sociedad Española de Enfermería Intensiva y Unidades Coronarias (SEEIUC). Published by Elsevier España, S.L.U. All rights reserved.
\end{abstract}

\section{¿Qué se conoce/qué aporta?}

Las enfermeras en cuidados intensivos desarrollan su labor profesional sin tener ningún requerimiento previo a la hora de optar a esos servicios. Las condiciones de los propios usuarios, su estado crítico y vulnerabilidad, sumado a las propias necesidades del soporte clínico hace que los profesionales deban afrontar una variabilidad muy grande de cuidados en poco espacio de tiempo. Esto ha generado que las propias sociedades científicas hayan intentado homogeneizar las competencias de las enfermeras pero no se ha recibido apoyo desde las instituciones.

\section{¿Implicaciones del estudio?}

Las propias enfermeras reconocen en el estudio que es necesario que se adopten medidas de formación específicas en las propias unidades ya que asumen que los conocimientos y habilidades que tenían cuando egresaron de la universidad eran insuficientes para lo que se encontraron de noveles. Y que aun siendo ya veteranas debe existir una formación que les mantenga actualizadas en todo momento con los nuevos avances y cuidados a aplicar.

\section{Introducción}

En las últimas décadas se ha producido un desarrollo importante en Enfermería, tanto en el ámbito académico como en el ámbito clínico. Los avances tecnológicos relacionados con la salud y el cuidado han provocado la necesidad de definir y adquirir nuevas competencias profesionales.

Los requisitos que actualmente rigen la mayor parte de los accesos al campo laboral son asimétricos, no reglados y fundamentados solo en la posesión de la titulación de Grado en Enfermería y en el tiempo trabajado previamente en un servicio determinado. Algunos estudios apoyan que la especialización en cuidados mejora los resultados en la atención, por tanto, se debe asegurar una formación acorde a determinados puestos laborales ${ }^{1}$.

Actualmente, en España, no existe una especialización oficial de Enfermería en cuidados al paciente crítico. Bien es verdad que existen universidades que sí dan formación en esta área como títulos propios a nivel de máster y experto universitario. En muchos países se están desarrollando programas formativos al respecto, sobre todo ante las evidencias demostradas por Aiken et al. ${ }^{2}$, los cuales encontraron relación entre los niveles educativos de las enfermeras y la mortalidad del paciente.

A pesar de que estamos en el Espacio Europeo de Educación Superior, las diferentes soluciones propuestas por Lakanmaa et al. ${ }^{3,4}$ desde Finlandia, no son simétricas a las 
que se pueden desarrollar en el Sistema Nacional de Salud Español. En el ámbito del cuidado al paciente crítico se han diseñado diferentes instrumentos de medida como el Intensive and Critical Care Nursing Competence Scale y el Basic Knowledge Assesment Tool, versión $7^{5}$, todos ellos surgidos a partir de las necesidades detectadas al respecto por la European Federation of Critical Care Nurses Association $(\mathrm{EfCCNa})^{6}$ para homogeneizar los cuidados intensivos en Europa. La necesidad de formación detectada no procede únicamente de las sociedades científicas, sino también de los profesionales, quienes observan carencias en formación e información en competencias específicas. En este sentido, se muestra la necesidad de colaborar para obtener mejores resultados y la inquietud por conseguir una formación óptima y ajustada a las exigencias del paciente y del entorno $\mathrm{UCl}$.

Por todo ello, se han creado diferentes respuestas a nivel europeo, todas ellas destinadas a la adquisición, mediante un programa formativo, de una serie de competencias que abarcan desde la faceta más técnica, a la conductual y emocional, haciendo hincapié en el proceso de comunicación. Se observa cómo la mejora de la formación de las enfermeras, y de los entornos donde se desarrolla la labor profesional, implica mejores resultados en los pacientes complejos ${ }^{7-9}$.

En España, han existido tentativas para el desarrollo de una acreditación, como un área de competencia específica o avanzada desde la Sociedad Española de Enfermería Intensiva y Unidades Coronarias, pero no ha llegado más allá de un intento aislado que no ha contado con el apoyo de las instituciones públicas ni privadas, que son las que finalmente realizan las contrataciones para dotación de enfermeras en las distintas unidades de cuidados intensivos ${ }^{10}$.

Aún en estos momentos, existe la necesidad de preguntarse, qué competencias son necesarias en cada unidad ${ }^{3}$, e incluso valorar qué demandan las propias enfermeras en el contexto laboral ${ }^{11-13}$, sin dejar atrás que la formación continua es un puente hacia la excelencia de los cuidados, más en unidades altamente tecnificadas donde los posibles riesgos también se asocian a una formación deficiente ${ }^{14,15}$. Las enfermeras como miembros del equipo multidisciplinar precisan una formación adecuada para hacer frente a los retos que plantea el paciente crítico y su entorno.

Partiendo de la base que la formación en cuidados avanzados es necesaria, nos planteamos, como objetivo de esta investigación, describir las necesidades formativas que detectan las enfermeras de $\mathrm{UCl}$ a través de sus vivencias y experiencias profesionales.

\section{Método}

Se planteó un diseño cualitativo descriptivo, con un enfoque fenomenológico. La fenomenología nos permite estudiar el fenómeno planteado a través de las experiencias vividas y el análisis de las necesidades de la realidad laboral en el ambiente cotidiano de una UCl. Este estudio se desarrolló con las enfermeras de la UCl de adultos del Hospital Universitario Insular de Gran Canaria, constituida como unidad polivalente de 30 camas.

La muestra a estudio se seleccionó, siguiendo un muestreo por conveniencia a partir de una plantilla de 72 enfermeras. Las participantes del estudio debían tener una experiencia en $\mathrm{UCl}$ igual o superior a tres años y colaborar en el estudio de forma voluntaria y desinteresada. La muestra fue de 15 participantes, cuya distribución se muestra en la tabla 1.

La técnica de obtención de información fue la entrevista semiestructurada que abordaba, con 57 preguntas abiertas, los ámbitos explorados y desarrollados por la $\mathrm{EffCNa}^{6}$ que son los siguientes: el clínico, profesional, gestión y educativo o de desarrollo. Además incluían las dimensiones del cuidado como eje transversal de estos ámbitos como son: cognitiva y del aprendizaje, técnica, integradora, relacional y moral-afectiva. Todas las preguntas se incluyeron mediante consenso del equipo investigador y apoyado en la bibliografía existente (tabla 2).

Tras el contacto personal con los participantes, se acordaron lugar, fecha y hora, para la realización de la entrevista. Las citas fueron flexibles con el ánimo de evitar contratiempos a los participantes. Al comienzo de la

Tabla 1 Datos sociodemográficos de los participantes

\begin{tabular}{|c|c|c|c|c|}
\hline Identificación & Sexo & Edad & Experiencia en $\mathrm{UCl}$ & $\begin{array}{l}\text { Experiencia total } \\
\text { como enfermera }\end{array}$ \\
\hline N1 & Hombre & 38 años & 14 años & 16 años \\
\hline N2 & Mujer & 39 años & 12 años & 16 años \\
\hline N3 & Mujer & 38 años & 8 años & 14 años \\
\hline N4 & Mujer & 31 años & 4 años & 8 años \\
\hline N5 & Mujer & 40 años & 16 años & 16 años \\
\hline N6 & Mujer & 37 años & 8 años & 16 años \\
\hline N7 & Hombre & 38 años & 13 años & 14 años \\
\hline N8 & Mujer & 37 años & 11 años & 15 años \\
\hline N9 & Mujer & 35 años & 13 años & 13 años \\
\hline N10 & Mujer & 37 años & 13 años & 15 años \\
\hline N11 & Hombre & 48 años & 14 años & 21 años \\
\hline N12 & Mujer & 37 años & 11 años & 16 años \\
\hline N13 & Mujer & 34 años & 10 años & 12 años \\
\hline N14 & Mujer & 39 años & 13 años & 14 años \\
\hline N15 & Mujer & 37 años & 11 años & 15 años \\
\hline
\end{tabular}




\begin{tabular}{|c|c|}
\hline Ámbito & Preguntas \\
\hline 1. Ámbito clínico & $\begin{array}{l}\text { ¿Qué conocimientos tiene que tener una enfermera de UCI para realizar un examen físico en el contexto del paciente crítico? } \\
\text { ¿Qué habilidades tiene que tener una enfermera de UCI para realizar un examen físico en el contexto del paciente crítico? } \\
\text { ¿Qué herramientas debe ser capaz de utilizar para poder obtener datos para la valoración? } \\
\text { ¿Qué aspectos emocionales tiene que tener en cuenta una enfermera de UCl para realizar un examen físico en el contexto del paciente } \\
\text { crítico? } \\
\text { ¿Qué aspectos personales tiene que tener en cuenta una enfermera de UCI para realizar un examen físico en el contexto del paciente } \\
\text { crítico? } \\
\text { ¿Qué aspectos sociales tiene que tener en cuenta una enfermera de UCI para realizar un examen físico en el contexto del paciente crítico? } \\
\text { Enumere los conocimientos y habilidades que tiene que tener la enfermera de cuidados intensivos para evaluar de manera crítica el plan } \\
\text { de cuidados } \\
\text { ¿Cómo puede la enfermera de UCI buscar la mejor evidencia científica en relación a los cuidados? } \\
\text { ¿Qué medidas debe conocer la enfermera a la hora de evaluar estrategias de mejora? } \\
\text { Priorice las patologías que debe conocer una enfermera de cuidados intensivos } \\
\text { Priorice los cuidados que debe conocer una enfermera de cuidados intensivos } \\
\text { Priorice las habilidades que debe tener una enfermera de cuidados intensivos en relación a los cuidados previamente citados }\end{array}$ \\
\hline 2. Ámbito profesional & $\begin{array}{l}\text { Enumere las fuentes en las que una enfermera puede buscar información para solucionar situaciones complejas } \\
\text { ¿Qué conocimientos tiene que tener la enfermera de UCI para poder hacer frente a decisiones complejas? } \\
\text { ¿Qué habilidades tiene que tener la enfermera de UCI para poder hacer frente a decisiones complejas? } \\
\text { ¿Cómo afronta una enfermera de UCI las decisiones de limitación del esfuerzo terapéutico? } \\
\text { ¿Qué habilidades tiene que tener una enfermera de UCl para afrontar la muerte de un paciente? } \\
\text { ¿La enfermera de cuidados intensivos, busca la excelencia a nivel clínico? } \\
\text { ¿Qué estrategias de comunicación debe saber utilizar una enfermera? } \\
\text { ¿Cómo se deben comunicar las enfermeras de UCl, en situaciones complicadas? } \\
\text { ¿Cómo debe ser la comunicación oral con el equipo? } \\
\text { ¿Cómo debería ser la información y comunicación en UCI? } \\
\text { ¿La comunicación escrita, con qué medidas debe fomentarse? } \\
\text { ¿Qué usos le debe dar la enfermera de UCl a las tecnologías de la información? }\end{array}$ \\
\hline
\end{tabular}


Tabla 2 (continuación)

\begin{tabular}{|c|c|}
\hline Ámbito & Preguntas \\
\hline 3. Ámbito de la gestión & $\begin{array}{l}\text { ¿En qué medida es necesario el seguimiento de los cuidados prestados? } \\
\text { ¿Qué medidas utiliza para garantizar una continuidad de los cuidados? } \\
\text { ¿Cuál es la importancia del liderazgo de las enfermeras en los equipos sanitarios? } \\
\text { ¿La coordinación del equipo sanitario, debe mantenerse? } \\
\text { ¿Las enfermeras recogen datos relacionados con los indicadores de calidad? } \\
\text { ¿Se hace un seguimiento para garantizar el cumplimiento de las actividades derivadas de los indicadores de calidad? } \\
\text { ¿En qué medida es capaz de delegar actividades la enfermera de UCI? } \\
\text { ¿Es capaz de planificar actuaciones a lo largo del tiempo? } \\
\text { ¿Gestiona adecuadamente su tiempo durante su horario laboral? } \\
\text { ¿Es capaz de gestionar y reevaluar las necesidades asistenciales a lo largo del turno de trabajo? } \\
\text { ¿Cómo debe gestionar los recursos de la manera más eficiente? } \\
\text { ¿Qué rol desempeña la enfermera de UCl como miembro de un grupo de trabajo? } \\
\text { ¿Hay un adecuado clima de trabajo, con transparencia, confianza y respeto? } \\
\text { ¿Qué medidas se pueden adoptar para que haya un adecuado clima laboral? } \\
\text { ¿Qué medidas debe tener un equipo de trabajo enfermero para tener una adecuada cohesión intragrupo? } \\
\text { ¿Se promueve el diálogo y la comunicación entre los miembros del equipo? } \\
\text { ¿Cómo se debe potenciar la seguridad clínica en el equipo asistencial? } \\
\text { ¿Qúmo están de involucradas las enfermeras en la seguridad del paciente? } \\
\text { ¿Qué medidas debe conocer una enfermera de UCl para prevenir y evitar eventos adversos? } \\
\text { ¿Son capaces los equipos asistenciales de trabajar bajo estrés? } \\
\text { ¿Se gestiona adecuadamente la carga de trabajo dentro de la unidad? } \\
\text { ¿Se posibilita la colaboración entre miembros del equipo? } \\
\text { ¿Cómo debe la enfermera de UCl ser capaz de adaptarse a los cambios en las necesidades, a las situaciones urgentes y emergentes? } \\
\text { ¿Cómo se deben potenciar las medidas de bienestar dentro de las Unidades de Cuidados Intensivos? } \\
\text { ¿Se fomentan prácticas para lograr un adecuado descanso de los pacientes? } \\
\text { ¿Qué medidas debe adoptar la enfermera para fomentar la orientación del paciente en tiempo y espacio? } \\
\text { ¿Se toman medidas para prevenir y/o evitar el delirio o la desorientación? }\end{array}$ \\
\hline $\begin{array}{l}\text { 4. Ámbito educativo y de } \\
\text { desarrollo }\end{array}$ & $\begin{array}{l}\text { ¿Qué actividades formativas realizan las enfermeras para estar adecuadamente formadas y mantener un desarrollo profesional } \\
\text { permanente? } \\
\text { ¿Tienen las actividades formativas repercusión a nivel clínico? } \\
\text { ¿Realiza la enfermera de UCl una formación reglada y relacionada con el ámbito de los cuidados intensivos? } \\
\text { ¿Qué medidas debe adoptar la enfermera a la hora de potenciar la investigación enfermera en su campo de actuación? } \\
\text { ¿Cómo colabora la institución en la formación continuada de las enfermeras de cuidados intensivos? }\end{array}$ \\
\hline
\end{tabular}


grabación se les explicaba el estudio, se les agradecía su participación y se les solicitaba que dieran su consentimiento verbal. También se recordaba que la entrevista estaba siendo grabada digitalmente y que las grabaciones, sin incluir datos personales, solo serían usados con fines de investigación en cumplimiento de la Declaración de Helsinki y de la Ley Orgánica 15/1999 de Protección de Datos de Carácter Personal y de su posterior actualización en la Ley Orgánica 3/2018.

El desarrollo total de las entrevistas tuvo una duración total de 17 horas 4 minutos y 16 segundos. Siendo el valor mínimo 50:26 y el máximo 1:44:25. Todas las entrevistas e incidencias fueron anotadas en el cuaderno de campo. El entrevistador era conocido por los entrevistados. Los investigadores, previo al inicio del proceso de la entrevista, consensuaron la no intervención en el discurso de los entrevistados, la no aportación de apreciaciones de carácter personal y la lectura de las preguntas a través de un mismo guión diseñado a tal fin.

El análisis de la información recogida se realizó siguiendo los datos propuestos por Cisterna-Cabrera ${ }^{16}$, un procedimiento de triangulación hermenéutica, «seleccionar la información obtenida en el trabajo de campo; triangular la información por cada estamento; triangular la información con los datos mediante los otros instrumentos y; triangular la información con el marco teórico».

La triangulación se llevó a cabo unificando previamente la información recogida de las grabaciones y el cuaderno de campo entre los 4 investigadores, siendo esta una fase de preparación. Además, se identificaron los ámbitos temáticos en los que se había basado la entrevista, facilitándosele a los investigadores un guión para identificar cada área temática. Cada uno de los investigadores, escuchó de manera personal todas las grabaciones y generó sus conclusiones personales adaptada a las áreas temáticas, codificando las respuestas tal y como también sugieren otros autores como Taylor y Bogdan ${ }^{17}$. Este hecho permitió crear categorías analíticas que, posteriormente, fueron analizadas mediante un procedimiento inferencial con conclusiones ascendentes, relacionado con áreas de consenso y divergencias entre los entrevistados. Se realizaron tres reuniones de consenso para validar las conclusiones de los investigadores centrándose en mantener la validez descriptiva de los discursos expresados, con la verificación de los discursos iniciales con las conclusiones de los investigadores. Indicando durante el desarrollo de las reuniones aquellos verbatims más pertinentes para valorar las necesidades que detectan las enfermeras en su formación en el área de cuidados intensivos, incluidos en cada categoría analítica. Las estrategias de consenso fueron la de validar la información recogida, su adaptación a la categoría incluida y su relectura una vez reorganizados para valorar que tuviera el mismo sentido que el participante había expresado.

Para poder trabajar con calidad y rigurosidad, se utilizó la reflexividad como un elemento analítico positivo identificando claramente, a lo largo del proceso del estudio, el rol del investigador y su influencia tanto en la recogida como en el análisis de datos. Los miembros del equipo investigador tuvieron en cuenta sus propias suposiciones internas y externas a la hora del análisis de datos con el objetivo de no provocar cambios en la información aportada por los participantes en la entrevista. El procedimiento de reflexividad ha permitido eliminar redundancias en los discursos, agrupar significados relevantes, poder transcribir literalmente en el texto de este manuscrito las experiencias más atingentes al objetivo del estudio, y lo que consideramos como más importante entender lo que está diciendo el entrevistado y no lo que esperábamos que dijera. El objetivo de este estudio es describir las necesidades formativas que detectan las enfermeras de $\mathrm{UCl}$ a través de sus vivencias y experiencias personales. El procedimiento de reflexividad permite no solo identificar las necesidades formativas de las enfermeras de $\mathrm{UCI}$ sino que facilita el encuadre de las citadas necesidades en el marco competencial descrito por la EfCCNa. La reflexividad nos permite cumplir el objetivo en el estudio. Este hecho nos permitió variar y modelar las conclusiones del equipo investigador. Para evaluar si la información era suficiente se valoró la saturación de datos y la validación por parte de los informantes durante el proceso de la entrevista.

Se respetó la confidencialidad de los informantes asignando durante la entrevista un código alfanumérico, en modo de $\mathrm{N} 1$ y sucesivos, no siendo identificados durante la entrevista.

Este estudio recibió la aprobación por parte de la Comisión Deontológica de la Universitat Jaume I. Las grabaciones y consentimientos serán custodiados por el investigador principal durante 5 años que es el tiempo de producción científica estipulado por la comisión y posteriormente serán destruidos.

\section{Resultados}

Para poder analizar adecuadamente la información se han utilizado las áreas de competencias descritas por la EfCCNa.

\section{Competencias del ámbito clínico}

A la hora de evaluar este ámbito, la experiencia de las enfermeras indica que se precisa un nivel de conocimientos muy elevado y una capacidad suficiente para aplicarlos de forma secuencial. Esto implica, disponer de suficientes destrezas que permitan la valoración, la aplicación de cuidados y el seguimiento del paciente. Por ello, según diferentes comentarios, la importancia de la observación de valores paramétricos como no paramétricos e ir desde la observación hasta la monitorización más avanzada hasta la fecha. Se hace especial hincapié en la monitorización y la aplicación de escalas, como paso previo a la hora de aplicar un razonamiento profesional destinado a llevar a cabo la aplicación de unos cuidados de calidad.

«Un conocimiento de qué se va a hacer y qué se está buscando, para poder aplicar los cuidados de manera eficiente (...) Una enfermera no puede llevar a cabo cuidados sin saber exactamente si convienen o no convienen en ese momento del proceso. Para realizar buenos cuidados hay que saber qué es lo que pasa» (N5).

«Se debe saber qué estás buscando, para saber qué encuentras y saber analizarlo, y para ello hay que tener tablas, experiencia y formación previa. Además de reevaluar, que para mí es lo más importante que tiene que estar continuamente haciendo una enfermera de $\mathrm{UCl}$ » (N9). 
«En UCl tienes que usar muchas herramientas que no te enseñan en la carrera, y es por ello que el personal de nueva incorporación puede ser uno de los sitios donde mayor desamparo y estrés puede tener a la hora de desarrollar su turno de trabajo (...) y esto se ve reforzado porque las patologías son muchas en $\mathrm{UCl}$, porque no suele haber un paciente que tenga una patología sola, y además debes saber cuidar desde una neumonía hasta un paludismo y aplicar los cuidados y controles adecuados» (N14).

Un aspecto destacado, es la necesidad de dar apoyo emocional y psicológico en este tipo de pacientes, desde la propia conciencia profesional de las circunstancias que envuelven este tipo de atención sanitaria, donde el ambiente hospitalario se torna especialmente hostil ante la gran cantidad de sonidos, ya sean alarmas, voces o el uso de tantos dispositivos que el usuario desconoce. Por ello, muchos entrevistados hablan de la importancia de la empatía y de reconocer cómo puede sentirse, tanto el usuario como los propios familiares, ante este entorno. Resulta llamativo también, cómo asumen que los aspectos del cuidado emocional o psicológico se suelen posponer, tal como ilustran:

«Quizás es un error por mi parte, pero priorizo estabilizar al paciente a conocer cuestiones emocionales» (N13).

«Al principio se deja un poco de lado el aspecto psicológico, porque no lo tratamos como algo urgente, cuando el paciente ya está estable y, generalmente, es un paciente de larga estancia, entonces sí nos volcamos en ese cuidado, pero al principio se deja un poco de lado» (N12).

Desde el punto de vista personal, los entrevistados reconocen que, al comienzo de su período laboral en $\mathrm{UCl}$, no se encontraban preparados para manejar emocionalmente ni de forma instrumental la presión derivada de la situación crítica que se vive en una UCI.

«Desde el minuto cero no tienes esas habilidades a no ser que hayas hecho unas buenas prácticas (...) y eso es una situación de estrés importante cuando se intenta una actividad laboral que en muchas veces tiene que ser exquisita» (N3).

«Todo lo necesario para cuidar al paciente crítico se adquiere, yendo a una UVI el primer día a trabajar sino teniendo una formación previa extensa (...) teniendo en cuenta que no todos los alumnos de enfermería pasan por la UVI y no todos desarrollan unas prácticas adecuadas para después enfrentarse al primer día que desde contratación te sueltan a llevar pacientes críticos, hay episodios de inseguridad y ansiedad» (N8).

A la hora de hablar sobre los cuidados que son indispensables, se enumeran muchísimos y de gran complejidad por parte de los entrevistados. Sí hay un consenso, en que lo importante es conocer el plan de cuidados de cada paciente, y ser capaz de llevarlo a cabo, teniendo en cuenta que:

«Las habilidades técnicas son importantes, pero son fundamentales la respuesta precoz ante complicaciones de las propias patologías, de los tratamientos y de los cuidados. Una enfermera de $\mathrm{UCl}$ debe ser muy reflexiva en lo que está pasando y lo que puede pasar, realizando una adecuada correlación de todos los datos de los que dispone» (N6).

«En el paciente crítico es importante saber priorizar (...) el enfermero de $\mathrm{UCl}$ debe adaptarse y, quizás, esta sea una de las características más importante del enfermero de intensivos» (N1).

Sí se observa cómo tanto las medidas de soporte, farmacológico o no, como las de monitorización, deben ser conocidas pormenorizadamente. Especial énfasis otorgan a saber manejar, vigilar y actuar con la ventilación mecánica. Surgiendo la entidad clínica en forma de dicotomía ¿qué hacer y qué no? Para lo cual, se debe fundamentar en una gran cantidad de competencias.

«Para mí es muy importante que no desarrolle úlceras por presión, pero esto choca también con la estabilidad hemodinámica o respiratoria» (N11).

«Adecuar el plan terapéutico-plan de cuidados a la realidad actual del usuario, es un reto continuo para las enfermeras» (N6).

\section{Competencias del ámbito profesional}

En el ámbito profesional, los entrevistados afirman la importancia de la formación continuada y de la capacidad de colaboración entre profesionales. La experiencia, entre las propias enfermeras, se torna como eje fundamental para solucionar situaciones complejas en los distintos turnos de trabajo.

«Consultar a compañeros o al farmacéutico, sobre cuestiones de administración de fármacos, siempre antes de provocar un error o evento adverso en el cuidado al paciente» (N2).

«Consulta a compañeros es la fuente fundamental, si después de esto no me quedo conforme después iría a buscar a Internet (...) pero generalmente las enfermeras experimentadas tienen la mayoría de las respuestas y suelen ser las más adecuadas, si no al médico de guardia. Además, alguna vez nos hemos visto buscando entre todos una solución» (N5).

Para poder trabajar con garantías, comentan que es necesaria una gran formación y especialización, reconociendo que los estudios de grado solo aportan destrezas y habilidades muy básicas para lo que el ambiente de UCI precisa. Y se reafirman en lo comentado en el ámbito clínico, además de añadir que:

«Todas las decisiones son complejas en cuidados intensivos, y esta razón es en la que se debiera basar que hay que tener una formación adecuada antes de trabajar en $\mathrm{UCl}$ ( $(\mathrm{N} 8)$.

«Aquí las enfermeras toman muchas decisiones, porque tenemos mucha autonomía» (N10).

En las UCl, la enfermeras hacen frente a situaciones, en el ámbito profesional, que repercuten sin duda alguna a nivel personal, como son la muerte de un paciente o las decisiones sobre limitación del esfuerzo terapéutico (LET). Asumen que es una situación muy personal y, en dos cosas sí se ponen 
de acuerdo, en que estas decisiones básicamente las acatan, considerándose un elemento pasivo y que llevarían peor emocionalmente el encarnizamiento terapéutico. Además, respecto a la muerte de los pacientes, hay casos en los que debido a las especiales características de esas personas les afectan más, sobre todo por la implicación personal que en el cuidado se asocia.

«Hablamos de un juicio médico (LET) en donde el enfermero no tiene otra opción de acatar la situación, aunque siempre se puede contrastar y verificar la información para que no haya ningún tipo de problema o información cruzada. Hay que asumirlo, acatarlo y reevaluar la situación adaptando el plan de cuidados» (N1).

«Personalmente no es tan duro para mí afrontar una limitación terapéutica que ves de manera clara y sí el ensañamiento que muchas veces se produce. Afronto peor la agonía del paciente» (N2).

Dentro del equipo asistencial, comentan vivir situaciones de estrés que afectan a la comunicación entre profesionales. Recomiendan aprender comunicación mediante simulacros y que esta tiene que ser clara, concisa y concreta, sin gritos y con datos objetivos, remarcando la comunicación bidireccional, fluida e integradora.

«Con voz pausada, y sin perder los papeles» (N13).

«que siempre sea claro el mensaje que quieres dar y a quién, y con el objetivo de solucionar la situación y no crear otro problema» (N4).

Además, se observan carencias en comunicación respecto a los familiares:

«No debemos olvidar que las enfermeras también tienen que informar y no sabemos informar sobre nuestro campo de actuación» (N15).

«Muchas veces y tras informarle el médico, los familiares salen corriendo buscando alguien que les explique cómo está su familiar y, generalmente, es la enfermera la que soluciona esas dudas y por eso es importante la comunicación dentro del equipo y dar a los familiares información veraz en todo momento» (N11).

\section{Competencias del ámbito de la gestión}

Muchos de los participantes ven en la continuidad de cuidados una de las grandes fortalezas de la $\mathrm{UCl}$, se vincula el seguimiento como una obligación del profesional que puede provocar una de sus mayores debilidades ya que su no cumplimiento genera la pérdida de esa fortaleza. La continuidad de cuidados se define como imprescindible. Como eje fundamental para conseguir este objetivo asistencial se consideran los registros de las actividades y las valoraciones de todos los profesionales incluidos en el cuidado del paciente. Además, otro elemento que potencia y asegura esa continuidad son las sesiones de enfermería que se realizan a primera hora del turno de mañana.

«El saber cómo están los usuarios se debe basar en información tanto escrita como verbal, además de contar con todos los miembros del equipo, otras enfermeras $\mathrm{y} / \mathrm{o}$ auxiliares que ya conocen la situación en la que está el paciente, o eventos que han pasado otros días» (N7).

En cuanto a la importancia del liderazgo de las enfermeras, creen que existe un liderazgo informal dentro del equipo sanitario.

«Las enfermeras no nos creemos la importancia que tenemos en una $\mathrm{UCl}$ y si nos lo creyéramos más, seríamos más líderes en más cosas» (N13).

En lo referente a la calidad en la gestión, comentan que se recogen indicadores, de calidad y que se considera como un elemento no fundamental de su labor diaria desaprovechando muchos datos e indicadores. Además lo desvirtúan como una cuestión externa a ellas como profesionales, recayendo tal responsabilidad, según los entrevistados, en el departamento de calidad.

«Se hace seguimiento de los indicadores de calidad cuando se puede y eso no suele pasar muy a menudo» (N5).

«Se desaprovechan muchos datos ya que vamos a la atención sanitaria inmediata y por eso dejamos estos datos de lado» (N1).

Afirman que además es necesario el trabajo en equipo, sobre todo a la hora de delegar actividades o usar los recursos humanos y materiales y reconocen que en la $\mathrm{UCl}$ se debe formar un equipo de trabajo cohesionado y responsable:

«las auxiliares de UCI hacen bastantes cosas que en planta no harían y eso es un valor dentro del equipo sanitario, aunque la enfermera tenga que estar allí continuamente» (N3).

«la enfermera tiene que estar atenta en todo momento, para poder hacer frente a todo lo que pasa» (N12).

«la confianza y el ambiente de trabajo tiene mucho que ver con las personas con las que trabajas, con su personalidad y, sobre todo, con su experiencia y habilidades, desde el médico hasta el celador» (N5).

«las enfermeras nuevas muchas veces son ninguneadas por el equipo médico, porque piensan que no son dignas de compartir el plan de cuidados y eso se puede convertir en una debilidad en la atención sanitaria» (N6).

A la hora de buscar situaciones de mejora vuelven a insistir en la necesidad de un aprendizaje gradual y reglado; manteniendo y reflexionando desde la gestión las decisiones tomadas, para que la seguridad clínica no se vea afectada.

«No se debe permitir que vengan 17 enfermeras de golpe sin experiencia a cubrir turnos, como ha pasado (...) mucha cantidad de personal nuevo provoca que el tema de la seguridad del paciente sin gente con experiencia sea un tema delicado» (N14).

«Las decisiones en la gestión nos han hecho formarnos y trabajar continuamente bajo estrés y no solo somos capaces de trabajar bajo estrés sino también somos especialistas (...) además hacerte cargo de otros compañeros nuevos que con voluntad intentan sacar el trabajo adelante pero requieren de un veterano que les guíe» (N6). 
«Asumir la carga asistencial, ser capaz de ayudar al compañero y concluir con éxito un turno de trabajo tiene mucho que ver con la formación y sobre todo con la experiencia que te da tablas para afrontar, casi todo lo que te echen» (N9).

\section{Competencias del ámbito educativo y de desarrollo}

En este ámbito se recalcan las ideas expresadas en los otros epígrafes, sobre la necesidad de que el personal de UCl esté continuamente en un proceso de formación continua, pero si se observa estos tres factores: la ausencia de requisitos previos, la falta de un plan de formación específico de $\mathrm{UCl}$ y la subjetividad de los intereses de cada enfermera se consideran debilidades que deben ser tratadas y mejoradas.

«cada unidad debería tener su formación específica con las necesidades detectadas y contar con sesiones propias dadas por expertos tanto a los nuevos como a los viejos» (N14).

«Hay que tener mucha voluntad, y sacar tiempo de tu propio tiempo personal para autoformarse» (N6).

«la institución colabora bastante poco pues hace docencia muy general y la $\mathrm{UCl}$ si tiene algo es que es muy específica en las necesidades formativas que requieren las enfermeras y todos los profesionales (...) aquí se usan mil cosas que no se usan en el resto del hospital» (N11).

Si la formación es difícil, en el contexto estudiado, la investigación se observa como algo secundario y nulamente valorado en el ambiente laboral, por lo que se debe incentivar las áreas de investigación mediante reconocimientos o becas.

«quizás estamos perdiendo gente muy válida, por no apostar por esta gente que investiga» (N12).

«la investigación de las enfermeras se ve como algo secundario y que no está incluido en su contrato con la empresa y por tanto solo investigan aquellas enfermeras muy motivadas y en su tiempo libre y eso es una pérdida para el Sistema» (N7).

\section{Discusión}

Si valoramos todos los ámbitos del presente artículo, de manera conjunta, podemos observar que los participantes hacen un énfasis importante en que la $\mathrm{UCl}$ es un servicio de gran complejidad, que sobrepasa cualquier formación pregrado recibida. En ese contexto, vinculan la experiencia como algo fundamental, pero asocian las necesidades de una formación muy extensa, para poder afrontar los retos asistenciales que se ven en el día a día. Además, existe inseguridad a la hora de abordar, por parte de las enfermeras noveles, determinados servicios como la $\mathrm{UCl}^{18,19}$.

Skees ${ }^{14}$ aborda la necesidad de desarrollar la educación continuada para alcanzar la excelencia, brindando a los profesionales la posibilidad de cuidar de manera segura, consiguiendo mejores resultados, ayudando a las enfermeras a incrementar su conocimiento y desarrollar su pensamiento crítico. Todo ello, se debe enfocar a las necesidades que se han detectado en las áreas específicas, tal y como nuestros participantes han expresado.

Se observa una falta de formación en determinados campos, que si se sigue lo estudiado por Gallaher ${ }^{20}$, se debe adaptar mediante un programa reglado, que se ciña a la mejora de la atención sanitaria. Detectando las áreas del cuidado que deben ser implementadas mediante programas educativos específicos. Se puede hacer frente a las necesidades encontradas mediante el uso de herramientas que ya han demostrado su utilidad, como sucede con la educación online ${ }^{21}$ para determinadas tipos de formaciones, ya que los propios entrevistados reconocen que las experiencias vividas en la propia UCI son muy didácticas.

Los participantes del presente estudio han observado la necesidad a su vez de tutelar, de manera progresiva, a las enfermeras noveles, y considerar la educación teóricopráctica con la ayuda de la simulación clínica, ya abordada por Thomason ${ }^{22}$, donde se destaca la preparación de enfermeras noveles con un programa completo y específico ${ }^{4,23}$, que debiera ser un objetivo de las propias unidades y del sistema sanitario con el fin de posibilitar que las enfermeras afronten los cuidados de una manera progresiva y segura. Hecho que fortalecería la asistencia sanitaria sobre todo en seguridad clínica, ya que como Ania-González et al. ${ }^{24}$ detectaron, las enfermeras tienen mejores conocimientos que los que demuestran en la práctica, en una técnica como la aspiración de secreciones, este hallazgo se puede extrapolar a otros cuidados. Lo dicho anteriormente, se puede usar tanto para las enfermeras noveles como para las veteranas con necesidades de reciclaje, o para el abordaje de nuevas terapias y cuidados, como han demostrado y nuestra muestra ha valorado como un punto de mejora, siempre se precisará una formación continua que posibilite una preparación eficiente en este tipos de áreas asistenciales ${ }^{25-27}$.

Como menciona Currey et al. ${ }^{26}$, el poder formarse es una oportunidad para lograr trabajar en equipo, hecho que se remarca como un dato a reforzar en los equipos asistenciales. Se observa en la literatura consultada, y también en la muestra, que cada día se adquieren nuevos roles y responsabilidades, lo que nos lleva a generar nuevas experiencias que varían el concepto de las enfermeras de $\mathrm{UCl}$, desde afrontar nuevas terapias y roles. Este hecho de adquisición de competencias en la asistencia se debe siempre dirigir al éxito de la asistencia, y esto depende mucho del desarrollo y habilidades de las enfermeras a la hora de cuidar al paciente crítico $^{2,26,28}$.

Actualmente se modifica el paradigma del cuidado del paciente crítico, ya que ahora se valora incluir a los familiares como elementos de éxito en el proceso de cuidar ${ }^{29}$, hecho que anteriormente no se consideraba una función primordial e incluso salir de las unidades y ampliar los cuidados enfermeros con otros roles que aseguren la continuidad del cuidado iniciado en $\mathrm{UCl}^{30}$. Estos cambios de actuaciones deben ir acompañados por una formación y unas guías de práctica clínica de consenso entre los miembros del equipo. Este hecho remarca la realidad, cada vez más acuciante, de que se adquieren más competencias en el ambiente clínico $^{13,31}$, y que la UCl es un ambiente en continua evolución científica ${ }^{32}$. Esta adquisición de conocimientos y esta evolución científica debe motivar una mayor implicación de las autoridades sanitarias ${ }^{33,34}$ para lograr políticas formativas, cada vez más centradas en lo que las enfermeras 
asistenciales demandan. En algunos estudios, incluso, se ha valorado el aspecto positivo que tiene una formación posgrado en esta área, tal y como observó Cotterill-Walker ${ }^{35}$ en su revisión bibliográfica, y que apoya los comentarios de los participantes en este estudio.

Teniendo en cuenta que se remarca mucho en este estudio, el trabajo en situaciones de estrés o las necesidades de cambio continuo debido a las necesidades propias de los pacientes críticos, se ha valorado muy positivamente la capacidad de priorización a la hora de aplicar cuidados por parte de las enfermeras. Se ha podido observar que es la comunicación ${ }^{36}$ de manera general, y específicamente en las situaciones complejas o críticas, un área fundamental que se tiene que desarrollar para no producir errores o lograr adecuados resultados, mediante dinámicas de grupo en los propios equipos asistenciales.

Además, reconocen nuestros entrevistados, que se hace difícil abordar las situaciones de pacientes que fallecen en las unidades, y que no todos los casos se viven de la misma manera. Observando que las estancias prolongadas ${ }^{37}$ conllevan una mejor aceptación de la limitación del esfuerzo terapéutico por la baja tasa de supervivencia. GálvezGonzález et al. ${ }^{38}$, en una muestra similar a la nuestra, afirman que las enfermeras requieren formación para poder compartir las decisiones del LET y así dejar de ser un ente pasivo en la toma de decisiones, pasividad que expresan también nuestras entrevistadas. Esta necesidad tiene que desarrollarse mediante la adquisición de competencias en este ámbito ${ }^{12}$.

En el ámbito de gestión, de formación y de desarrollo, las enfermeras consultadas reconocen que es necesario tomar decisiones que orienten tanto la carga de trabajo de la unidad, como el plan formativo de las enfermeras, para mejorar las áreas de trabajo ${ }^{39}$ y la acogida de nuevo personal, de una manera progresiva y efectiva ${ }^{40-42}$. Todo ello generará unos mejores resultados en salud ${ }^{2,7,8}$, una menor cantidad de errores en la asistencia sanitaria ${ }^{20}$. En un contexto global, se debería extender cómo la autoevaluación de los propios profesionales nos pueden llevar a áreas de mejora en las distintas competencias enfermeras que se requieren en el día a día de una $\mathrm{UCl}^{43}$.

\section{Conclusión}

La muestra estudiada hace especial hincapié en que se requieren sólidos conocimientos y destrezas a la hora de poder cuidar de una manera efectiva a un usuario en la unidad de cuidados intensivos. En este aspecto, reconocen que es necesario estar formándose continuamente, y que con los conocimientos que tenían al egresar de los estudios universitarios no eran suficientes para cuidar de una manera adecuada a los pacientes críticos. Han necesitado de vivir múltiples experiencias, y realizar formación ex profeso para poder hacerlo de una manera efectiva.

El ámbito clínico, relacionado con el cuidado al paciente crítico, ha sido asociado con una necesidad de formación ajustada a la realidad del entorno $\mathrm{UCl}$ y a las necesidades que presentan distintos tipos de pacientes. Partiendo de unas competencias generales aplicables a todos los pacientes críticos, se destaca la importancia de profundizar en competencias más específicas que aseguren los cuidados en casos más concretos.

En el ámbito de la gestión, se observan tres necesidades: gestionar adecuadamente el personal y la carga de trabajo, el uso de materiales de manera eficiente y el liderazgo de las enfermeras. Se destaca el equipo multidisciplinar como eje fundamental que debe ser promovido y apoyado tanto por los profesionales como por los gestores.

Por todo ello, se debe proponer la creación de estudios centrados en las competencias a desarrollar en cada una de las unidades de $\mathrm{UCl}$, saber qué se requiere de una enfermera, y asegurar una competencia en ese campo. Además, tener como objetivo, el no hacer solamente, sino hacer de la manera más excelente posible.

\section{Conflicto de intereses}

Los autores declaran no tener ningún conflicto de intereses.

\section{Bibliografía}

1. Tourangeau AE, Giovannetty P, Tu J, Wood M. Nursing-related determinants of 30-day mortality for hospitalized patients. Can J Nurs Res. 2002;33:71-88.

2. Aiken LH, Clarke SP, Cheung RB, Sloane DM, Silber JH. Educational levels of hospital nurses and surgical patient mortality. JAMA. 2003;290:1617-23.

3. Lakanmaa RL, Suominen T, Perttilä J, Puukka P, Leino-Kilpi $\mathrm{H}$. Competence requirements in intensive and critical care nursing-still in need of definition? A Delphi study. Intensive Crit Care Nurs. 2012;28:329-36.

4. Lakanmaa RL, Suominen $T$, Perttilä J, Ritmala-Castrèn $M$, Vahlberg T, Leino-Kilpi H. Graduating nursing studentsbasic competence in intensive and critical care nursing. J Clin Nurs. 2014;23(5-6):645-53.

5. Toth J. Development of the Basic Knowledge Assessment Tool for Medical-Surgical Nursing (MED-SURG BKAT)৫ and implications for in-service educators and managers. Nurs Forum. 2011;46:110-6.

6. European Federation of Critical Care Nursing associations (EfCCNa). Declaración de consenso en Educación de Postgrado para Enfermería de Cuidados Intensivos en Europa [internet]. 2004. [consultado 14 Mar 2018]. Disponible en: http://www.seeiuc.com/efccna/educaposgrado.pdf.

7. Stimpfel AW, Sloane DM, McHugh MD, Aiken LH. Hospitals Known for Nursing Excellence Associated with Better Hospital Experience for Patients. Health Serv Res. 2016;51:1120-34.

8. Silber JH, Rosenbaum PR, McHugh MD, Ludwig JM, Smith HL, Niknam BA, et al. Comparison of the Value of Nursing Work Environments in Hospitals Across Different Levels of Patient Risk. JAMA Surg. 2016;151:527-36.

9. McHugh MD, Rochman MF, Sloane DM, Berg RA, Mancini ME, Nadkarni VM, et al. Better Nurse Staffing and Nurse Work Environments Associated With Increased Survival of In-Hospital Cardiac Arrest Patients. Med Care. 2016;54:74-80.

10. Pascual J, Bueno P, Cuenca M, Asiain MC, Marín B. Certificación de la competencia profesional de la enfermera de cuidados críticos: encuesta de opinión. Enferm Intensiva. 1998;9:16-20.

11. Square ND. Modeling clinical applications in intensive care settings for nursing orientation. Adv Neonatal Care. 2010;10:325-9.

12. White KR, Roczen ML, Coyne PJ, Wiencek C. Acute and critical care nursesṕerceptions of palliative care competencies: a pilot study. J Contin Educ Nurs. 2014;45:265-77. 
13. Lindberg E. Competence in critical care: what it is and how to gain it: qualitative study from staff's point of view. Dimens Crit Care Nurs. 2006;25:77-81.

14. Skees J. Continuing education: a bridge to excellence in critical care nursing. Crit Care Nurs Q. 2010;33:104-16.

15. Melnyk BM, Fineout-Overholt E. Evidence-based practice in nursing and healthcare: a guide to best practice. $2 .{ }^{\mathrm{a}}$ ed Philadelphia: Wolters Kluwer/Lippincott Williams \& Wilkins; 2011.

16. Cisterna-Cabrera, F. Categorización y triangulación como procesos de validación del conocimiento en investigación cualitativa. Theoria [Internet]. 2005 [consultado 26 Jun 2018]; 14:61-71. Disponible en: https://www.redalyc. org/articulo.oa?id=29900107.

17. Taylor S, Bogdan R. El trabajo con los datos. Análisis de los datos en investigación cualitativa. En: Paidós, editor: introducción a los métodos cualitativos de investigación. $3^{\mathrm{a}}$ ed. Barcelona; 2000. p. 152-76.

18. Marrero González CM. Estudio fenomenológico de la experiencia de incorporación al contexto laboral de enfermeras egresadas de la universidad de La Laguna entre 2009 y 2014. [tesis doctoral en Internet]. [La Laguna]: Universidad de la Laguna; 2017 [consultado 20 Jun 2018]. Disponible en: http://riull.ull.es/xmlui/handle/915/4485.

19. Marrero González CM, García Hernández AM. Narraciones pertenecientes a enfermeras egresadas de la universidad de La Laguna sobre cómo mejorar su incorporación al mundo laboral en Tenerife. Ene. [Internet]. 2017 [consultado 27 Jun 2018];11(2). Disponible en: http: //scielo.isciii.es/scielo.php?script=sci_arttext\&pid=S1988$348 \times 2017000200004 \& \operatorname{lng}=e s$.

20. Gallagher L. Continuing education in nursing: a concept analysis. Nurse Educ Today. 2007;27:466-73

21. Sweeney NM, Saarmann L, Flagg J, Seidman R. The keys to successful online continuing education programs for nurses. J Contin Educ Nurs. 2008;39:34-41.

22. Thomason TR. ICU nursing orientation and postorientation practices: a national survey. Crit Care Nurs Q. 2006;29:237-45.

23. Kaihlanen AM, Lakanmaa RL, Salminen L. The transition from nursing student to registered nurse: the mentor's possibilities to act as a supporter. Nurse Educ Pract. 2013;13:418-22.

24. Ania-González N, Martínez-Mingo A, Eseberri-Sagardoy M, Margall-Coscojuela MA, Asiain-Erro MC. Evaluación de la competencia práctica y de los conocimientos científicos de enfermeras de $\mathrm{UCl}$ en la aspiración endotraqueal de secreciones. Enferm Intensiva. 2004;15:101-11.

25. Stinson KJ. Nursesáttitudes, clinical experience, and practice issues with the use of physical retraints in critical care units. Am J Crit Care. 2016;25:21-6.

26. Currey J, Eustace P, Oldland E, Glanville D, Story I. Developing professional attributes in critical care nurses using Team-Based Learning. Nurse Educ Pract. 2015;15:232-8.

27. Currey J, Oldland E, Considine J, Glanville D, Story I. Evaluation of postgraduate critical care nursing students' attitudes to, and engagement with, Team-Based Learning: a descriptive study. Intensive Crit Care Nurs. 2015;31:19-28.

28. Sørensen D, Frederiksen K, Grøfte T, Lomborg K. Practical wisdom: a qualitative study of the care and management of non-invasive ventilation patients by experienced intensive care nurses. Intensive Crit Care Nurs. 2013;29:174-81.
29. Buckley P, Andrews T. Intensive care nurses' knowledge of critical care family needs. Intensive Crit Care Nurs. 2011;27: 263-72.

30. Dawson D, McEwen A. Critical care without walls: The role of the nurse consultant in critical care. Intensive Crit Care Nurs. 2005;21:334-43.

31. Goldman HG. Role expansion in intensive care: survey of nurses' views. Intensive Crit Care Nurs. 1999;15:313-23.

32. Camelo SH. Professional competences of nurse to work in Intensive Care Units: an integrative review. Rev Lat Am Enfermagem. 2012;20:192-200.

33. Ministerio de Sanidad Política Social. Gobierno de España. Unidad de Cuidados Intensivos. Estándares y recomendaciones. [Internet]. Madrid. 2010 [consultado 10 May 2018]. Disponible en: http://www.mscbs.gob.es/ organizacion/sns/planCalidadSNS/docs/UCl.pdf.

34. Uhrenfeldt L, Lakanmaa RL, Flinkman M, Basto ML, Attree M. Collaboration: a SWOT analysis of the process of conducting a review of nursing workforce policies in five European countries. J Nurs Manag. 2014;22:485-98.

35. Cotterill-Walker SM. Where is the evidence that master's level nursing education makes a difference to patient care? A literature review. Nurse Educ Today. 2012;32:57-64.

36. Sánchez Sánchez F, Sánchez Marín FJ, López Benavente Y. Un estudio cualitativo para conocer las características que definen y mejoran la formación en comunicación asistencial e interprofesional. Rev Calidad Asistencial. 2008;23:253-8.

37. Santana Cabrera L, Sánchez-Palacios M, Hernández Medina E, Eugenio Robaina P, Villanueva-Hernández A. Characteristics and prognosis of patients with very long stay in an Intensive Care Unit. Med Intensiva. 2008;32:157-62.

38. Gálvez González M, Ríos Gallego F, Fernández Vargas L, del Águila Hidalgo B, Muñumel Alameda G, Fernández Luque C. El final de la vida en la Unidad de Cuidados Intensivos desde la perspectiva enfermera: un estudio fenomenológico. Enferm Intensiva[Internet]. 2011[consultado 10 May 2018];22:13-21. Disponible en: https://www.elsevier.es/es-revista-enfermeriaintensiva-142-pdf-S1130239910001124.

39. American Association of Critical-Care Nurses. AACN standards for establishing and sustaining healthy work environments: a journey to excellence. Am J Crit Care. 2005;14:187-97.

40. Juers A, Wheeler M, Pascoe H, Gregory N, Steers C. Transition to intensive care nursing: a state-wide, workplace centred program-12 years on. Aust Crit Care. 2012;25:91-9.

41. Boyle M, Butcher R, Conyers V, Kendrick T, MacNamara M, Lang S. Transition to intensive care nursing: establishing a starting point. Aust Crit Care. 2008;21:190-8.

42. Roselló Hervas M, Valls Andrés S. Determinación del perfil de enfermería en las unidades de cuidados intensivos cardiológicos según sus competencias. Enferm Cardiol [Internet]. 2012 [consultado 22 May 2018];57:51-58. Disponible en: https: / /www.enfermeriaencardiologia.com/wpcontent/uploads/57_06.pdf.

43. Asiain MC, Margall MA. Autoevaluación de la competencia profesional de enfermeras asistenciales. En: Investén - isciii. VIII Encuentro de Investigación en Enfermería. Sevilla: Edita Unidad de coordinación y desarrollo de la Investigación en Enfermería; 2004. p. $140-3$. 